INPLASY

PROTOCOL

To cite: Yi et al. Efficacy and Safety of pediatric massage in the treatment of Anorexia: a protocol for systematic review and meta-analysis. Inplasy protocol 202130050. doi: 10.37766/inplasy2021.3.0050

Received: 15 March 2021

Published: 15 March 2021

Corresponding author: Baoxiu Yi

1285070461@qq.com

Author Affiliation: Jiangxi University of Traditional Chinese Medicine

Support: Jiangxi province.

Review Stage at time of this submission: The review has not yet started.

Conflicts of interest: None delcared.

\section{Efficacy and Safety of pediatric massage in the treatment of Anorexia: a protocol for systematic review and meta-analysis}

Yi, BX1; Chen, WG²; Deng, G3; Wang, YY4; Wang, JF5; Chi, ZH6.

Review question / Objective: A systematic review and metaanalysis of clinical randomized controlled studies on pediatric massage for Anorexia following the rules of evidence-based medicine.

Condition being studied: Anorexia is a common and frequent disease in clinical pediatrics. It refers to a chronic digestive disorder syndrome with loss of appetite or disappearance and reduced food intake. The incidence of anorexia in children is very high, if not timely, safe and effective treatment, will have a huge impact on the growth and development of children. The toxic side effects of related treatment drugs often worry clinicians. Pediatric massage is external therapy, with green, safe and effective characteristics, lack of evidence-based medicine evidence support. A systematic evaluation and meta analysis of the safety and efficacy of pediatric massage in the treatment of anorexia will be carried out in this paper to provide a powerful Evidence.

INPLASY registration number: This protocol was registered with the International Platform of Registered Systematic Review and Meta-Analysis Protocols (INPLASY) on 15 March 2021 and was last updated on 15 March 2021 (registration number INPLASY202130050).

\section{INTRODUCTION}

Review question / Objective: A systematic review and meta-analysis of clinical randomized controlled studies on pediatric massage for Anorexia following the rules of evidence-based medicine.
Condition being studied: Anorexia is a common and frequent disease in clinical pediatrics. It refers to a chronic digestive disorder syndrome with loss of appetite or disappearance and reduced food intake. The incidence of anorexia in children is very high, if not timely, safe and effective treatment, will have a huge impact on the growth and development of children. The 
toxic side effects of related treatment drugs often worry clinicians. Pediatric massage is external therapy, with green, safe and effective characteristics, lack of evidence-based medicine evidence support. A systematic evaluation and meta analysis of the safety and efficacy of pediatric massage in the treatment of anorexia will be carried out in this paper to provide a powerful Evidence.

\section{METHODS}

Search strategy: We'll retrieve eight electronic databases, including the PubMed、Embase、Cochrane Library, Web Of Science、Chinese Biomedical Literature Database (CBM), China National Knowledge Infrastructure (CNKI), Wanfang Database (WF), China Science Journal Database (VIP), the retrieval date was established from the database to March 2021.

Participant or population: There are clear and recognized diagnostic and curative criteria, and all patients are diagnosed with anorexia in children, regardless of sex, age and source of cases.

Intervention: Pediatric massage will include all different schools of pediatric massage techniques. Mixed therapy based on pediatric massage will also be included.

Comparator: The control group will receive one of the following treatments: routine pharmaceutical treatment, no treatment, and placebo.

Study designs to be included: Clinical randomized controlled trials (RCT) containing pediatric massage for anorexia will be included, but do not limit language and publication status.

Eligibility criteria: Reported in chinese and english, and meet the "PICOS", will be considered for inclusion in this overview.

Information sources: We'll retrieve eight electronic databases, including the PubMed、Embase、Cochrane Library, Web
Of Science、Chinese Biomedical Literature Database (CBM), China National Knowledge Infrastructure (CNKI), Wanfang Database (WF), China Science Journal Database (VIP), the retrieval date was established from the database to March 2021.

Main outcome(s): Clinical efficacy, including total effective rate or cure rate, clinical symptom score, will be considered as the primary outcome.

Additional outcome(s): Body mass index (BMI), TCM symptom score changes and recurrence will be secondary outcomes.

Data management: Two reviewers will independently extract relevant data from the eligible RCTs, including the first author, participants' baseline characteristics, sample size, intervention, intervention time, follow-up, results, and adverse events. Any discrepancies will be resolved through consultation with a third reviewer. If necessary, we will also contact the original author for more information.

Quality assessment / Risk of bias analysis: Two evaluators independently select the literature according to the inclusion and exclusion criteria and cross-check. In case of disagreement, a third evaluator will assist in the decision. The extracted data included the first author, year of publication, number of patients, age, gender, intervention measures, outcome indicators, etc. The Jadad scale to evaluate quality into literature, including: random sequence (right 2 points, 1 points not clear, inappropriate 0), distribution, hidden (right 2 points, 1 points not clear, inappropriate 0 ), blinded (right 2 points, 1 points not clear, inappropriate 0 ), lost to follow-up and exit (describe 1 points, not describe 0 ); $0-3$ is classified as low quality and $4-7$ as high quality.

Strategy of data synthesis: We will use RevMan V.5.3 software for statistical analysis. For continuous variables, when outcomes were measured by the same scale, the results were reported as 
standardized mean difference (MD) and 95\% confidence interval ( $\mathrm{CI})$; when different scales were used, the results were reported as standardized mean difference (SMD) and $95 \% \mathrm{Cl}$. Categorical data will be calculated with the risk ratio (RR) and $95 \%$ CI.

Subgroup analysis: If significant heterogeneity is detected in our metaanalysis, we will perform subgroup analysis based on different control groups.

Sensitivity analysis: When there are sufficient RCTs, we will conduct sensitivity analysis based on methodological quality, sample size and missing data to evaluate the robustness of the research results.

Language: No limitation of language.

Country(ies) involved: China.

Keywords: Pediatric massage; anorexia; TCM therapy; protocol; systematic review and meta analysis.

Contributions of each author:

Author 1 - Baoxiu Yi - The author drafted the manuscript.

Email: 1285070461@qq.com

Author 2 - Wenguang Chen - The author provided statistical expertise.

Email: 2291545079@qq.com

Author 3 - Gen Deng - The author contributed to the development of the selection criteria, and the risk of bias assessment strategy.

Email: 1084053154@qq.com

Author 4 - Yiyi Wang - The author read, provided feedback and approved the final manuscript.

Email: 1554255919@qq.com

Author 5 - Jinfeng Wang - The author read, provided feedback and approved the final manuscript.

Email: 1151885392@qq.com

Author 6 - Zhenhai Chi - The author provided statistical expertise.

Email: 348916661@qq.com 